\title{
Forderungen des djb an die Bundesjustizministerin/ den Bundesjustizminister und die Justizministerinnen und -minister der Länder
}

1. Das Modell des Einheitsjuristen soll unbedingt beibehalten werden, weil nur so der hohe Standard der hiesigen Juristinnen- und Juristenausbildung gewahrt bleibt und die erforderliche Durchlässigkeit zwischen den juristischen Tätigkeitsfeldern gegeben ist.

2. Der djb begrüßt grundsätzlich die Umstellung des rechtswissenschaftlichen Studiums auf das Bachelor/ Master-System.

Wünschenswert ist die Aufteilung in ein dreijähriges Grundlagenstudium, dessen erfolgreicher Abschluss zum Führen des Titels „Bachelor im Recht“ berechtigt und die Absolventinnen und Absolventen zur Ausübung aller juristischen Berufe auf Sachbearbeiterebene befähigt. Der erfolgreiche Abschluss dient als Zugangsvoraussetzung für ein abschließendes zweijähriges Vertiefungsstudium zum Erwerb des „Master im Recht“, dessen erfolgreicher Abschluss zur Ausübung aller hochqualifizierten juristischen Berufe auf höherer Ebene, mit Ausnahme der reglementierten, befähigt.

Der Titel „Master im Recht“ ist notenunabhängige Zugangsvoraussetzung zum fakultativ sich anschließenden zweijährigen Referendariat, dessen erfolgreicher Abschluss zur Ausübung der reglementierten Berufe berechtigt. Die Entkoppelung von bestimmten Notenvorgaben gewährleistet die Etablierung des „Master im Recht" als vollwertigen Hochschulabschluss.

3. Inhaltlich sollte im Pflichtfachbereich ein Studienfach „Familien- und Gleichstellungsrecht“ angeboten werden. Wichtig ist darüber hinaus die Verstärkung der Vermittlung der für das Berufsleben unentbehrlichen so genannten „soft skills“. Insbesondere bieten sich die jeweiligen Arbeitsgemeinschaften dazu an, den Umgang mit Parteien und Anwältinnen oder Anwälten, Streitschlichtung und Mediation sowie Kostenmanagement, Personalführung und Arbeitsorganisation zu trainieren. Stärker als bisher muss auch die Beurteilung komplexer wirtschaftlicher Sachverhalte als Basis juristischer Entscheidungsfindung vermittelt werden. Geschlechterfragen sind als Querschnittsthema in alle Fächer einzubeziehen und mit ihren rechtlichen Inhalten zu behandeln, wo es die unterschiedlichen Lebensrealitäten von Frauen und Männern erfordern.
4. Ziel eines jeden Ausbildungsabschnittes, der auf den Erwerb eines berufsqualifizierenden Titels zielt, muss die Berufsfähigkeit der Absolventinnen und Absolventen sein, nicht die Berufsfertigkeit.

5. Zwingend erforderlich ist ein System kontinuierlicher Abschichtung in allen Ausbildungsphasen.

Im gesamten Studium sind Abschlussklausuren an jedem Semesterende sowie im Bachelorstudium die Einführung einer Zwischenprüfung nach dem vierten Semester wünschenswert. Im Referendariat sollten die schriftlichen Examensprüfungen nach jeder Ausbildungsstation im jeweiligen Fach stattfinden und sich nach Abschluss der Anwaltsstation am Ende eine mündliche Prüfung anschließen.

6. Die einzelnen Klausurleistungen in den Ausbildungsabschnitten sind auf die Endnote anzurechnen. Unbedingt erforderlich ist auch die angemessene Einbeziehung der Bewertungen der praktischen Ausbilderinnen und Ausbilder in die Endnote.

7. Der djb fordert eine kontinuierliche Qualitätssicherung der gesamten Ausbildung.

Im Studium kann diesen hohen Qualitätsanforderungen durch Einübung juristischer Kompetenzen in Kleingruppen und Seminaren entsprochen werden. Im Referendariat sind regelmäßige Fortbildungsmaßnahmen für die Ausbilderinnen und Ausbilder zwingend. Auch die angemessene Bezahlung der Arbeitsgemeinschaftsleiterinnen und -leiter und eine Einordnung als hauptamtliche Tätigkeit bei richterlichen Ausbilderinnen und Ausbildern wirkt qualitätssichernd.

Die Zuständigkeit zur Ausgestaltung der Anwaltsstation einschließlich der begleitenden Arbeitsgemeinschaften sollte bei den Anwaltskammern liegen, um eine praxisnahe, sach- und fachgerechte Vorbereitung auf den Anwaltsberuf zu gewährleisten. Wünschenswert ist die Schaffung bundeseinheitlicher Standards durch gesetzliche Vorgaben.

Unverzichtbar zur Qualitätssicherung ist die Beibehaltung der Unabhängigkeit der Justizprüfungsämter. 